\title{
Metacercaria : \\ Proctoeces progeneticus (Trematoda Digenea) \\ chez une Gibbula (Gastropoda Prosobrancbiata) de la côte atlantique du Maroc.
}

\section{Observations sur la famille Fellodistomatidae}

\author{
par Robert-Ph. DOLLFUS
}

\section{Résumé}

Plusieurs fois, des métacercaires progénétiques de Proctoecinae ont été trouvées chez des Lamellibranches. Dans le cas présent, l'hôte est un Gastropode du genre Gibbula.

Le genre Proctoeces est conservé ici dans la famille Fellodistomatidae, bien qu'une famille Proctoecidae ait été proposée.

Je considère que, dans chaque sous-famille de Fellodistomatidae, il y a un seul type de cercaire, sauf dans la sous-famille Steingophorinae, où il y a des cercaires de deux types. Les cercaires sont leptocerques ou trichocerques chez les Steringophorinae; elles sont seulement furcocerques, ou seulement microcerques, ou seulement anoures, dans les autres sous-familles.

\section{DESCRIPTION}

Corps subcylindrique, environ 3 à 5 fois plus long que large, à contour de lagéniforme à cylindriforme en extension sur le vivant; ovale, allongé au repos, atténué antérieurement, élargi à la fin du deuxième tiers de sa longueur ; extrémités arrondies.

Ventouse orale terminale profonde. Ventouse ventrale plus grande que l'orale, très profonde en vue sagittale; son centre est situé vers la mi-longueur du corps en extension et vers la fin du premier tiers au repos.

Cuticule non spinulée, lisse ou un peu striée transversalement.

Prépharynx présent, mais observable seulement sur coupes sagittales. Le pharynx est à peu près aussi large que long et un peu rétréci antérieurement. Esophage court, 
mais pouvant atteindre en extension la longueur du pharynx. Branches intestinales divergeant un peu en avant du bord antérieur de la ventouse ventrale, s'étendant longitudinalement en direction postérieure, se terminant sans atteindre l'extrémité postérieure du corps.

Testicules plus ou moins ovales, à bord entier, disposés généralement en tandem, parfois presque au même niveau, occupant chacun, en largeur, environ la moitié de l'espace intercaecal, en partie en avant, en partie en arrière de la limite entre les deux derniers tiers de la longueur du corps. Lorsque le testicule antérieur est du côté droit, le postérieur est du côté gauche. Le testicule antérieur, l'ovaire et la poche du cirre sont toujours du même côté. Les vasa efferentia se réunissent tout près de l'extrémité postérieure de la poche du cirre.

La poche du cirre, très grande, plus ou moins arquée, est située soit dorsalement, soit latéralement à la ventouse ventrale, tantôt du côté droit, tantôt du côté gauche ; si elle est latérale, elle vient en contact de l'intestin ; antérieurement, elle s'étend jusqu'à la mi-largeur du corps, mais, postérieurement, elle s'étend moins loin vers la ligne médiane. L'extrémité postérieure de la poche du cirre est occupée par la vésicule séminale interne, tubuleuse, formant des sinuosités (1). La pars prostatica qui lui fait suite est très développée, elle se continue par le canal éjaculateur qui passe dans le cirre et aboutit à un sinus génital extrêmement réduit. Le cirre proprement dit est très court. Près de l'extrémité du canal éjaculateur se trouve une protubérance musculaire (2) bien développée, pouvant faire saillie dans le très court sinus génital et au dehors par pression sur le vivant (fig. 2-3).

Le pore génital est médian ou submédian, ventralement à la bifurcation de l'intestin, contre le bord antérieur de la ventouse ventrale.

L'ovaire est généralement un peu plus petit que les testicules, sa forme est sphérique ou ovale, à bords entiers ; cependant, chez un de mes exemplaires, l'ovaire était trilobé. Il est situé très peu en arrière de la ventouse ventrale et même il atteint généralement le bord postérieur de celle-ci. Sa position est un peu latérale ou submédiane, du côté droit si la poche du cirre est à droite, du côté gauche si elle est à gauche (situs inversus). Il n'y a qu'un très petit espace entre l'ovaire et 'e testicule antérieur et celuici est toujours du même côté que l'ovaire quand ce dernier est un peu latéral.

Il n'y a pas de receptaculum seminis, mais il y a un canal de Laurer s'ouvrant dorsalement, presque sur la ligne médiane, au niveau de l'espace entre l'ovaire et le testicule antérieur. L'oviducte reçoit le vitelloducte impair, très court. Le vitelloducte transverse, mal distinct, passe entre l'ovaire et le testicule antérieur. Les follicules vitellogènes sont très petits et très peu nombreux, ils se présentent sous la forme de courtes files

(1) Il ne semble pas y avoir de vésicule séminale externe; cependant, chez quelques individus, il y a, contre le fond de la poche du cirre, un petit amas de granulations qui peut donner l'impression de représenter une vésicule séminale externe, c'est, apparemment, une petite dilatation du court canal déférent.

(2) Au sujet de cette protubérance musculaire des Proctoeces qui a reçu diverses appellations, voir Ts. Fujita $(1925$, p. 53, fig. 6 \& crête fibreuse du pénis »), S. Yamaguti (1934, p. 411, fig. 79 « large muscular nodule », 1938, p. 103), R. F. H. Freeman et J. Llewellyn (1958, p. 442, 443, fig. 1 et pl. I, fig. 3 « muscular papille »). 


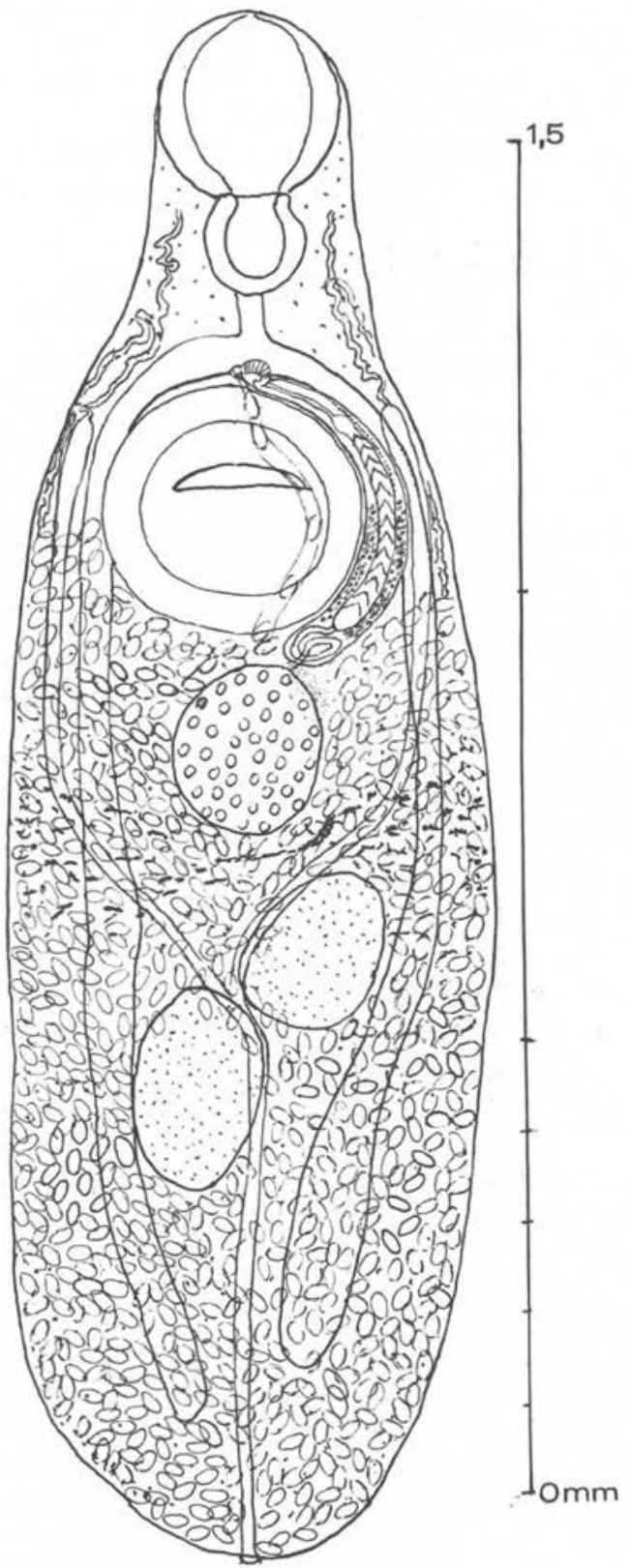

Fig. 1. - Métacercaria : Proctoeces progeneticus n. sp. D'après le vivant et une préparation colorée. La région antérieure du corps est en contraction, la région postérieure en extension. 

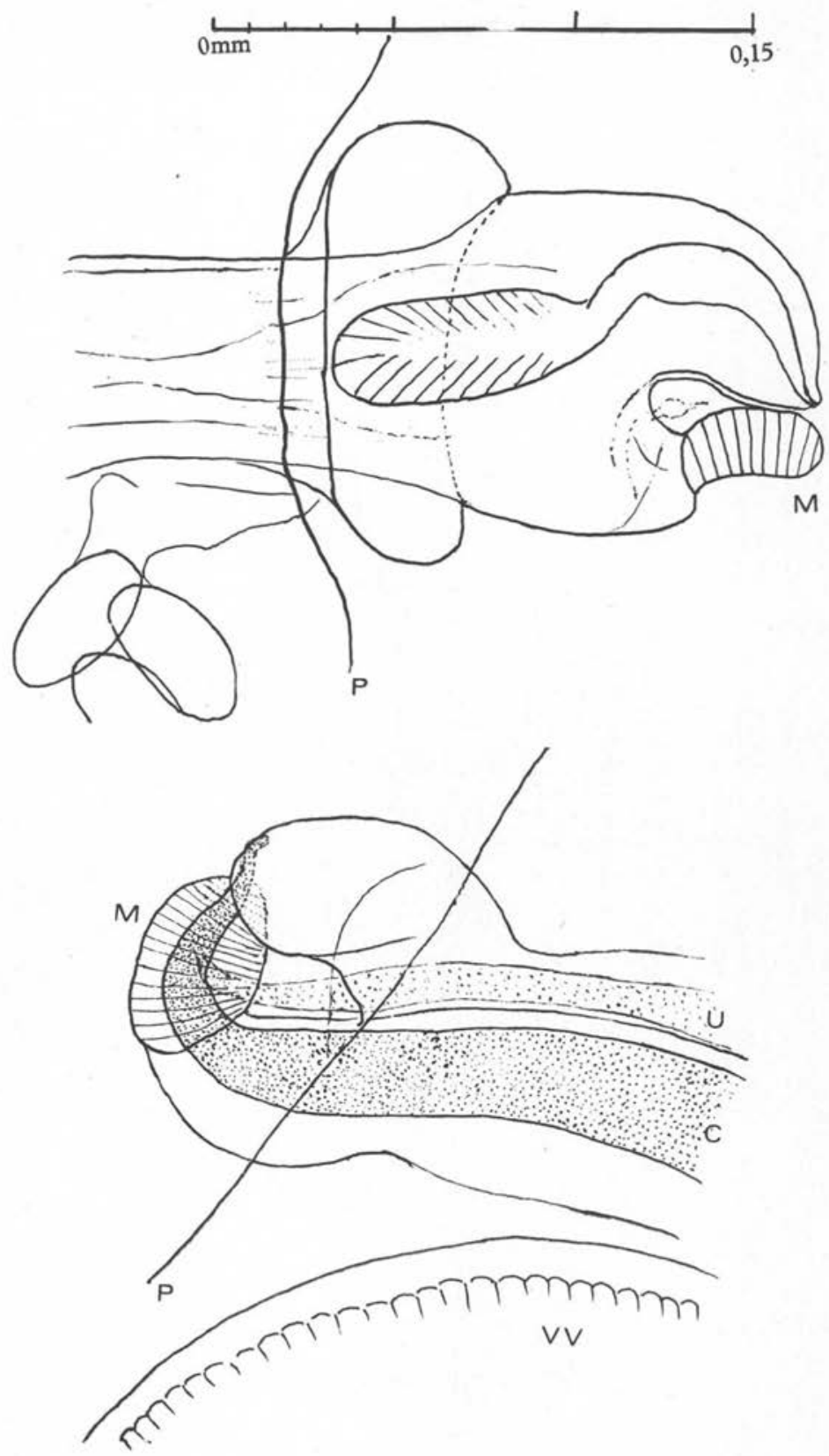

Fig. 2 et 3. - Protubérance musculaire du cirre telle qu'elle apparaît chez des individus déprimés sous lamelle. M Protubérance musculaire, C canal éjaculateur, U terminaison de l'utérus, V V ventouse ventrale, $\mathbf{P}$ paroi du corps 
linéaires transversales dans la zone ovario-testiculaire. Il y a, dans tout le parenchyme du corps, de nombreuses cellules glandulaires qu'il ne faut pas confondre avec des follicules vitellogènes (cf. R. Ph. Dolifus in Ts. Fujita 1925, p. 54, note 1).

L'utérus, partant de l'ootype, se dirige d'abord postérieurement, ventralement aux testicules, puis s'étend du côté droit au côté gauche et de la face dorsale à la face ventrale, la branche ascendante croisant la branche descendante. Il remplit presque la totalité du corps en arrière de la ventouse ventrale, jusqu'au pore excréteur.

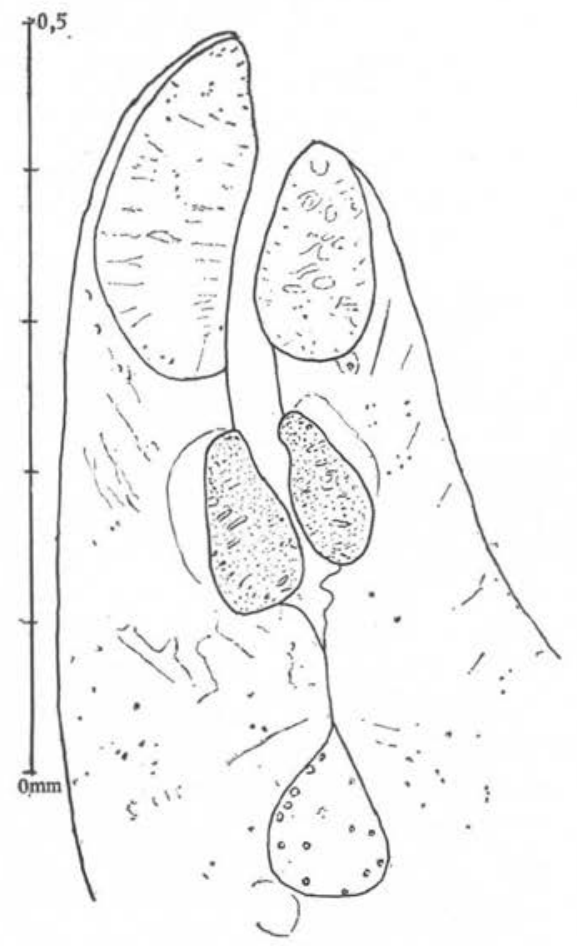

FıG. 4. - Coupe sagittale de l'extrémité antérieure pour montrer l'existence du prépharynx, invisible sur les spécimens vus par les faces

Les œufs, excessivement nombreux, sont ovales, à petit opercule et mesurent, à maturité, sur le vivant, dans la partie terminale de l'utérus : $49,5 \times 29,7$ à $51 \times 26,4 \mu$. Au voisinage du pore génital, on remarque que les æufs sont embryonnés et contiennent un miracidium cilié (3).

(3) Chez les Proctoeces, la production d'œufs est souvent abondante et précoce. Chez maculatus, dit T. Odhner (1911, p. 108), les individus de $1 \mathrm{~mm}$ sont déjà remplis d'œufs. Le miracidium mobile dans les œufs a été observé par J. R. Uzmann (1953, p. 449), chez la métacercaire progénétique qu'il a découverte chez Mytilus edulis L., de même par R. F.H. Freeman et J. Llewellyn (1958, p. 445, pl. II, fig. 7) chez leur métacercaire progénétique du rein de Scrobicularia plana (Da Costa) (1 à 13 métacercaires par hôte).

Toutes les espèces de métacercaires progénétiques n'ont pas de nombreux cufs ; un petit nombre d'œufs seulement a été observé par Ts. Fujita chez sa métacercaire parasite de la gonade d'Ostrea gigas Thunberg (1 à 10 métacercaires par hôte). 
La vessie excrétrice est en Y. Chez les individus dont le tiers postérieur est en extension, le tronc impair est plus long que les branches et la bifurcation est à peu près au niveau de l'espace intertesticulaire. Les branches se dirigent latéralement puis longitudinalement, ventralement à l'intestin ; elles atteignent le niveau du bord antérieur de la ventouse ventrale, ou même le niveau de l'œsophage.

Dimensions $(\mathrm{mm})$ de trois individus un peu contractés ; montés dans le baume du Canada après coloration :

\begin{tabular}{|c|c|c|c|}
\hline Longueur & 1,541 & 1,634 & 1,742 \\
\hline Largeur $\ldots \ldots \ldots \ldots \ldots \ldots \ldots$ & 0,670 & 0,804 & 0,670 \\
\hline Ventouse orale $\ldots \ldots \ldots \ldots \ldots$ & 0,241 & 0,227 & 0,174 \\
\hline Pharynx $\ldots \ldots \ldots \ldots \ldots \ldots$ & $0,120 \times 0,120$ & $0,147 \times 0,134$ & $0,134 \times 0,13$ \\
\hline Esophage $\ldots \ldots \ldots \ldots \ldots \ldots$ & 0,051 & 0,053 & 0,027 \\
\hline Ventouse ventrale $\ldots \ldots \ldots \ldots \ldots$ & $0,268 \times 0,348$ & $0,268 \times 0,348$ & $0,268 \times 0,33$ \\
\hline Ovaire $\ldots \ldots \ldots \ldots \ldots \ldots \ldots$ & $0,160 \times 0,201$ & 0,201 & 0,201 \\
\hline Testicule antérieur $\ldots \ldots \ldots \ldots$. & $0,308 \times 0,187$ & 0,174 & $0,201 \times 0,147$ \\
\hline Testicule postérieur $\ldots \ldots \ldots \ldots$. & $0,281 \times 0,201$ & 0,2 & $0,227 \times 0,1$ \\
\hline Poche du cirre, longueur ....... & 0,435 & 0.316 & 0,462 \\
\hline diamètre $\quad \ldots \ldots \ldots \ldots \ldots \ldots \ldots \ldots$ & 0,079 & 0,085 & 0,066 \\
\hline
\end{tabular}

En extension maximum sur le vivant, la longueur peut atteindre $3 \mathrm{~mm}$. Les plus petits immatures mesuraient $0,459 \times 0,263 \mathrm{~mm}$.

\section{LOCALISATION DANS L'HOTE}

J'ai trouvé cette métacercaire progénétique chez $90 \%$ des Gibbula umbilicalis (Da Costa) du rivage de Témara-Gayvil'e (sud de Rabat, Maroc) (4), en mai 1964. Elle est libre dans les tissus, invariablement entre la radula et l'intestin ; il y en a généralement une ou deux dans chaque Gibbula, mais on peut en trouver exceptionnellement jusqu'à cinq. Les immatures sont rares, presque tous les individus sont bourrés d'œufs. C'est une larve extrêmement mobile, s'étirant et se rétractant avec vivacité, ce qui rend son étude sur le vivant difficile, la position des organes les' uns par rapport aux autres se modifiant sans cesse.

\section{IDENTIFICATION SPECIFIQUE}

La distinction entre les espèces du genre est difficile et les auteurs ne sont pas d'accord sur les synonymies. Ben Dawes (1946, p. 245 ; 1947, p. 266) a considéré erythraeus comme un synonyme de maculatus. Pour M. L. Hanson (1950, p. 83) et pour H. W. Manter (1954, p. 528), erythraeus est synonyme de subtenuis, de même pour K. I. Skrjabin et V. Koval (1957, p. 396). Pour R. F. H. Freeman et J. Llewellyn (1958, p. 450), c'est à subtenuis que se rapportent erythraeus et magnorus; mais pour $\mathrm{H}$. W. Stunkard et J. R. Uzmann (1959, p. 193) c'est à maculatus que se rapporte subtenuis et, pour H. W. Manter et M. Hanson Pritchard (1962, p. 115) on doit rapporter à maculatus à la fois subtenuis et erythraeus.

(4) Je dois particulièrement remercier Mlle Hélène Gantès, qui a pris la peine de récolter les Gibbula. 
Le statut spécifique des espèces maculatus, erythraeus, subtenuis, insolitus, ostreae, major, magnorus, macrovitellus a été longuement discuté par R. F. H. Freeman et J. Llewellyn (1958, p. 447-451) et je n'ai pas l'intention de reprendre ici la discussion. Peu de caractères considérés comme distinctifs semblent constants.

Mes spécimens parasites de Gibbula ont le pore génital médian ou submédian, alors qu'il est nettement déplacé vers la gauche «etwas nach links » chez le spécimen de maculatus figuré par Looss (1901, p. 403, fig. 1), mais, chez les spécimens rapportés à maculatus par Wlassenko (1931, p. 100, fig. 6) il est à peu près médian. H. W. Stunkard et J. R. Uzmann (1959, p. 186-187, fig. 3-4) le figurent beaucoup plus latéral que Looss pour maculatus, de même que V. Koval (in Skrjabin et Koval 1957, p. 391, fig. 114 a), mais il est figuré moins latéral par Freeman et Llewellyn (1958, p. 443, fig. 1) chez la métacercaire progénétique qu'ils considèrent comme subtenuis.

Est-ce bien maculatus qui a été étudié sous ce nom par Stunkard et Uzmann? C'est assez difficile à admettre et l'on remarque, chez les spécimens figurés par ces auteurs, que la partie antérieure du tube digestif, en avant de la bifurcation, partie qui comprend l'œsophage + le début de l'intestin, a une forme triangulaire, étendue à la fois en longueur et en largeur, qui n'existe ni chez les spécimens rapportés à maculatus par d'autres descripteurs ni chez mes spécimens parasites de Gibbula, en outre, le sinus génital est beaucoup plus court que chez maculatus.

La présence ou l'absence d'un prépharynx sont peut-être utilisables pour aider à caractériser spécifiquement quelques formes, mais en contraction, le prépharynx n'apparaît pas, c’est ainsi que, pour ostreae, Fujita ne l'a pas figuré, mais chez un spécimen original que j'ai examiné (voir R. Ph. Dollfus 1925, p. 50, note 2), il est très nettement présent. Un prépharynx est figuré par R. F. H. Freeman et J. Llewellyn (1958, p. 443, fig. 1) pour la métacercaire qu'ils ont rapportée à subtenuis.

Chez mes spécimens parasites de Gibbula, le prépharynx, invisible sur le vivant, est observable seulement sur des coupes sagittales, il est long de $35,5 \mu$.

La forme des testicules n'apporte aucun caractère utilisable, de même celle de l'ovaire. Chez major, l'ovaire est représenté et décrit par S. Yamaguti (1934, p. 411, fig. 79) comme trilobé, mais il ne semble pas que ce caractère soit discriminatif ; Wlassenko (1931, p. 101) a relaté qu'un de ses exemplaires de maculatus avait l'ovaire trilobé.

Peut-on tirer parti des dimensions des œufs ? C'est incertain car les dimensions données par les descripteurs ne concernent pas toujours des œufs normaux à maturité et il y a une variation très étendue chez un même individu. On a mesuré des œufs dont la longueur atteignait sur le vivant $79 \mu$ (Odhner 1911, p. 108) chez maculatus, aussi semble-t-il difficile de rapporter à la même espèce des formes dont les œufs sont longs de 40 à $50 \mu$ ou plus petits.

Après comparaison de mes spécimens parasites de Gibbula avec les descriptions publiées, je suis amené à les considérer comme appartenant à une espèce nouvelle, progeneticus, caractérisée comme il suit :

Prépharynx visible seulement sur coupes sagittales. Court œsophage. Poche du cirre beaucoup plus longue que le diamètre longitudinal de la ventouse ventrale. Pore 
génital médian ou submédian, au niveau de la bifurcation intestinale, souvent contre le bord antérieur de la ventouse ventrale. Sinus génital extrêmement réduit. Ovaire et testicules à peu près de mêmes dimensions, ceux-ci généralement disposés en tandem, rarement tous les deux au même niveau. Ovaire médian ou un peu déplacé vers la droite ou vers la gauche ; s'il est vers la gauche, la poche du cirre est vers la gauche, le testicule antérieur vers la gauche. Situs inversus chez environ la moitié des individus. Vitellogènes très peu développés.

Eufs extrêmement nombreux, contenant, à maturité, un miracidium cilié, mobile.

Parasite de Gibbuia umbilicalis (Da Costa), où il est libre entre l'intestin et la radula.

Comme on le voit dans le tableau, plusieurs fois des Proctoeces au stade métacercaire ont été rencontrés chez des Mollusques marins. Jusqu’à présent, ces Mollusques étaient seulement des Pélécypodes. Il est vraisemblab'e qu'il existe des Proctoeces à cycle évolutif abrégé. Si l'espèce étudiée par Stunkard et Uzmann est maculatus, comme l'admettent ces auteurs, maculatus est une espèce à deux cycles, l'un abrégé, sans poisson hôte définitif, l'autre normal avec adulte dans la partie postérieure du tube digestif (*) d'un Té'éostéen mangeur de Mollusques. Ce serait aussi le cas de subtenuis, si l'on admet que c'est bien cette espèce qui a été étudiée par Freeman et Llewellyn.

\section{POSITION SYSTEMATIQUE DE PROCTOECES}

T. Odhner (1911, p. 107-108, diagnose)

Pour Odhner (1911, p. 106) il s'agit, comme pour Tergestia, d'un genre aberrant de la famille Steringophoridae T. Odhner (1911, p. 97-98, diagnose de la famille); «aberrant» parce que quelques-uns de ses caractères ne s'accordent pas avec la diagnose de la famille. La possibilité d'admettre dans une famille des genres, tels que Proctoeces, dont les caractères ne s'accordent que partiellement avec la diagnose de la famille a été commentée par F. Poche (1919, p. 314) qui l'a estimée légitime.

Comme nom pour cette famille, T. Odhner (1911, p. 98, note 5) a dit qu'il préférait Steringophoridae à Fellodistomidae; il rejeta de même Fellodistominae au profit de Steringophorinae. Ainsi que je l'ai rappelé (1952, p. 372), comme il existait déjà une sous-famille Fellodistominae W. Nicoll 1909, antérieure à Steringophorinae T. Odhner 1911, celle-ci tomba en synonymie de celle-là et Fellodistomidae, devenu Fellodistomatidae, a prévalu.

J'ai admis (1952, p. 373-377) la famille Fellodistomatidae (W. Nicoll 1909, T. Odhner 1911) avec quatre sous-familles: 1) Fellodistomatinae (W. Nicoll 1909) emendata, pour les genres où l'utérus est seulement prétesticulaire (**); 2) Steringophori-

(*) C'est en raison de cette localisation intestinale que le genre a été nommé par Odhner

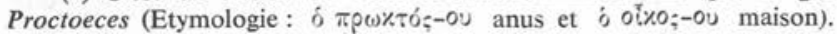

(***) C'est pourquoi je ne considère pas Fellodistomum saviniense V. Dyk et Dykova, 1964 (de la vésicule et de l'intestin de Trutta trutta morpha fario L. et Thymallus thymallus (L.), en Tchécoslovaquie) comme appartenant "aux Fellodistomatinae; l'utérus s'étendant en arrière des testicules. 
nae T. Odhner 1911, emendata, pour les genres où l'utérus s'étend en arrière des testicules ; 3) Monascinae R. Ph. Dollfus 1948 (= Haplocladinae T. Odhner 1911) pour les genres où l'utérus est à la fois prétesticulaire et posttesticulaire ; 4) Discogasteroidinae H. D. Srivastava 1939, pour des genres où l'utérus est aussi à la fois prétesticulaire et posttesticulaire, mais avec divers autres caractères ne permettant pas l'admission dans les Monascinae.

Pour W. Nicoll (1915, p. 345), Proctoeces appartient aux Haplocladinae (= Monascinae). J'ai accepté (1952, p. 376). cette attribution, mais K. I. Skrjabin et Véra Koval (1957, p. 387, 388 diagnoses) ont proposé, dans les Fellodistomatidae, une sous-famille particulière: Proctoecinae K. I. Skrjabin et Véra Koval, qui est devenue la famille Proctoecidae Ed. Caballero y C. (1959, p. 38-39, diagnose de la famille). A mon avis, le seul argument qui pourrait justifier une famille particulière, ce serait une morphologie de la cercaire complètement différente de celle des cercaires des autres Fellodistomatidae; il en sera question plus loin (page 769).

Une position systématique très différente est celle innovée par S. Yamaguti (1958, p. 34-35) qui a placé Proctoeces dans la sous-famille Heterorchiinae R. Ph. Dollfus 1950, fondée pour Heterorchis H. A. Baylis 1915, genre dont les affinités sont incertaines et encore discutées (cf. R. Ph. Dollfus 1950, p. 40, note 1), mais que je ne crois pas possible d'admettre dans les Fellodistomatidae, ni même dans la superfamille Fellodistomatoidea G. R. La Rue 1957.

Caballero (1959) a mis en synonymie de Proctoecidae, la famille Xenoperidae F. Poche 1926, p. 194-196. Cette dernière famille a été fondée sur le genre Xenopera W. Nicoll (1915, p. 34) et Poche l'a placée dans les Hemiurida. Cette attribution a été catégoriquement rejetée par T. Odhner (1927, p. E, note 2), les affinités avec Proctoeces n'étant pas douteuses et Odhner a rappelé que, déjà antérieurement (1911, p. 107), il avait placé Proctoeces, comme genre aberrant, dans sa famille Steringophoridae (c'est-à-dire Fellodistomatidae).

La famille Xenoperidae F. Poche a été retenue comme valable par K. I. Skrjabin et Guschanskaja $(1958$, p. 816-817, 933) et ces auteurs l'ont admise dans le sous-ordre Azygiata G. R. La Rue 1957, ce qui ne pouvait pas être accepté.

En 1952 (p. 376, note 18), j’ai dit que Xenopera était évidemment synonyme de Proctoeces, parce que, à mon avis, les différences entre Proctoeces et Xenopera me paraissaient d'ordre spécifique ou, tout au plus, subgénériques, n'ayant pas une importance justifiant un genre séparé. C'est aussi l'opinion de R. F. H. Freeman et J. Llewellyn (1958, p. 449) qui ont discuté la valeur des différences entre Proctoeces insolitus (W. Nicoll) et Pr. subtenuis (Linton) ; chez l'espèce du Queensland, le canal hermaphrodite prolongeant la poche du cirre s'étend postérieurement jusqu'à une petite distance en arrière de la ventouse ventra'e et la poche du cirre se trouve en position longitudinale, entièrement en arrière de la ventouse ventrale; en outre, il y a une vésicule séminale externe. T. Odhner (1911, p. 107, note 15) a estimé que c'était par suite d'une pression que, dans la figure donnée par Looss de maculatus, la poche du cirre s'était déplacée jusqu'en arrière de la ventouse ventrale et que, par suite, le sinus 


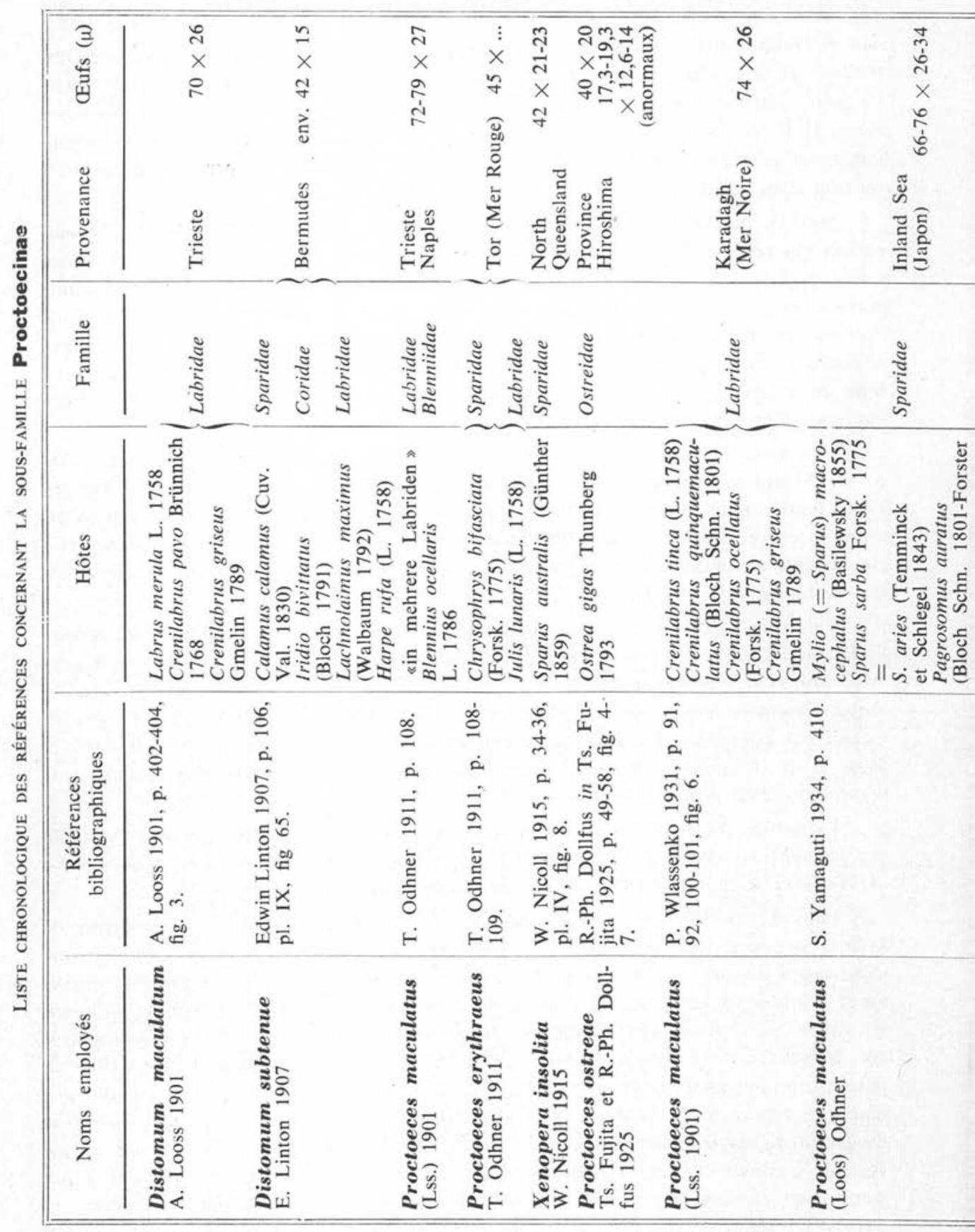




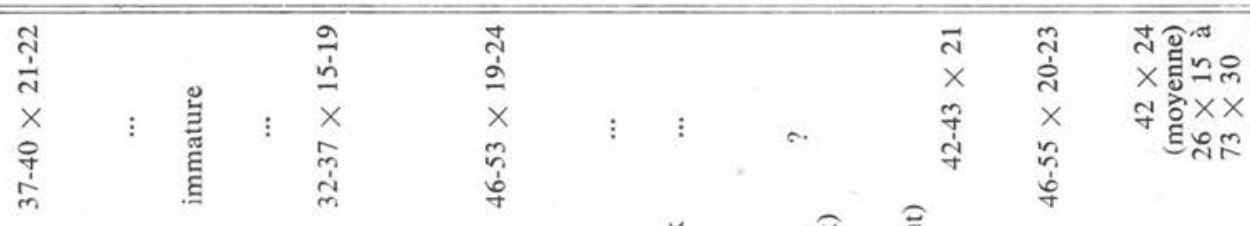

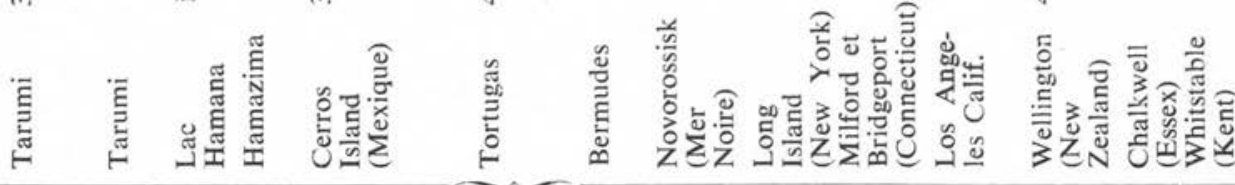

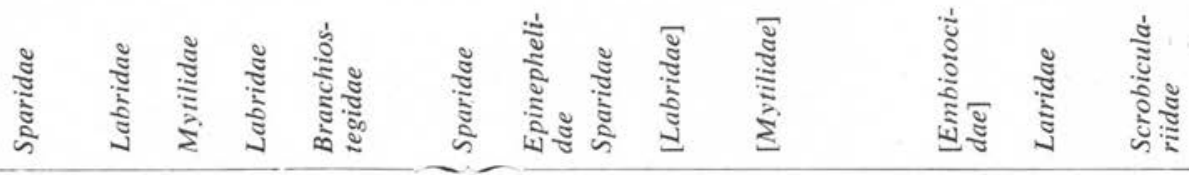

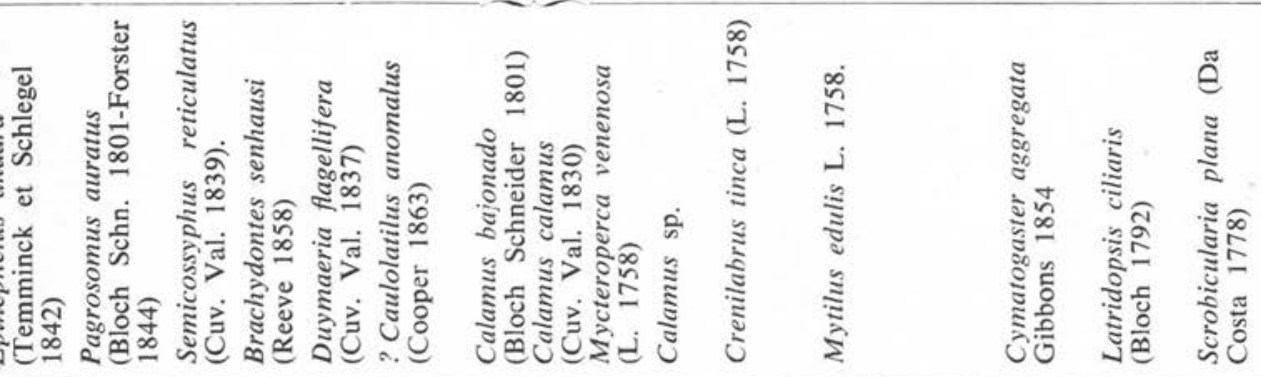

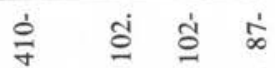

$\therefore$ - क व

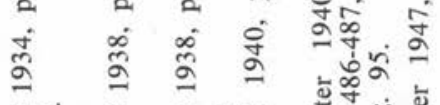

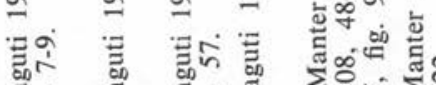

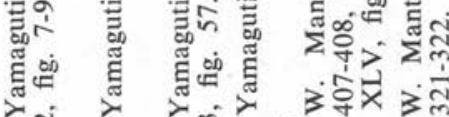

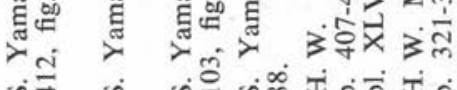

कि

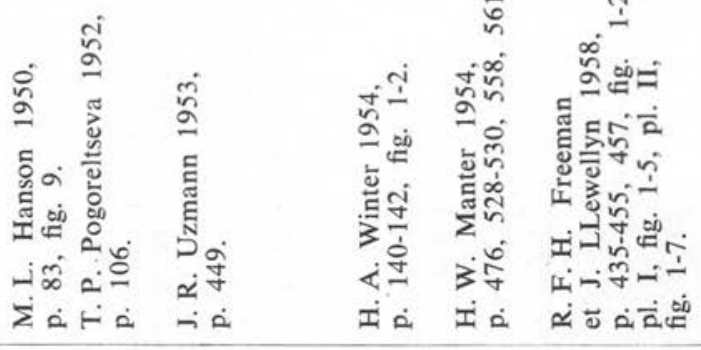

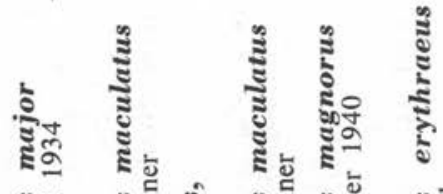

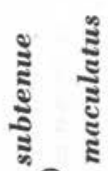

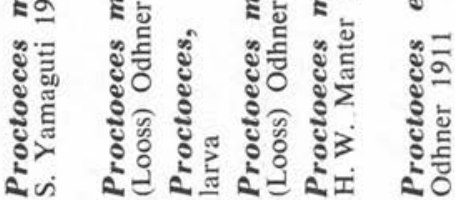

縞

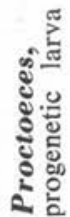

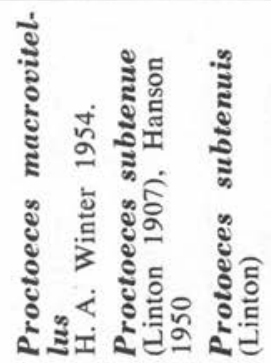




\begin{tabular}{|c|c|}
\hline \multicolumn{2}{|r|}{ 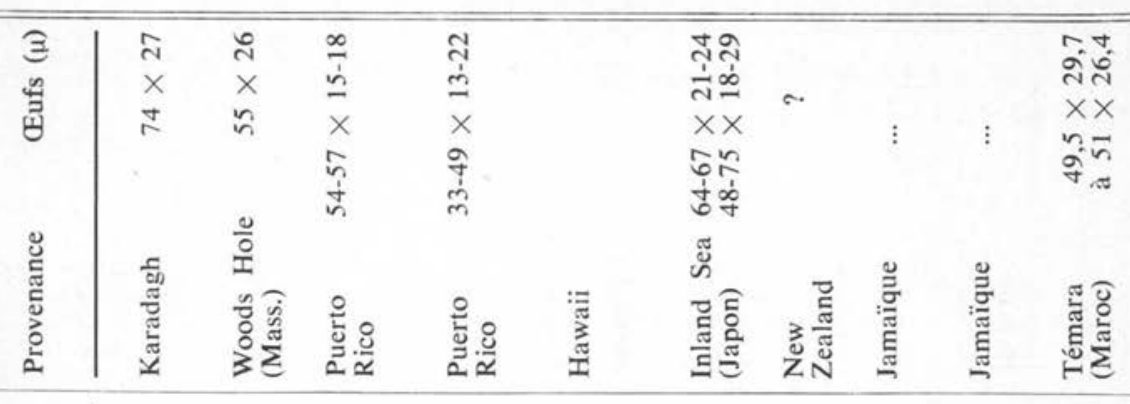 } \\
\hline 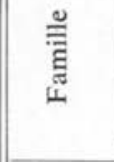 & 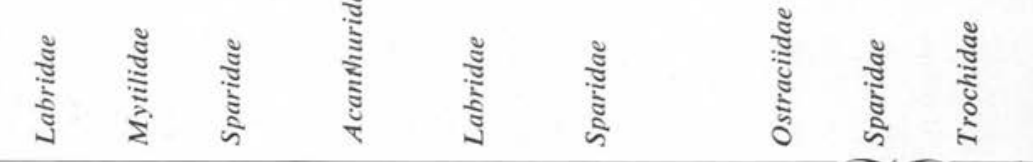 \\
\hline 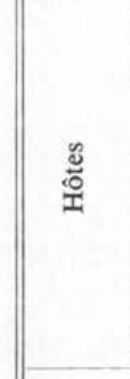 & 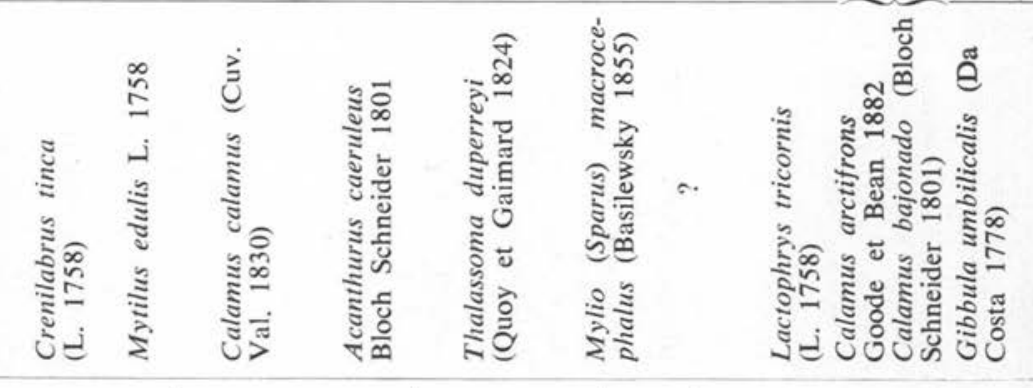 \\
\hline 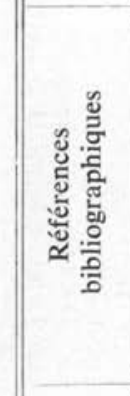 & 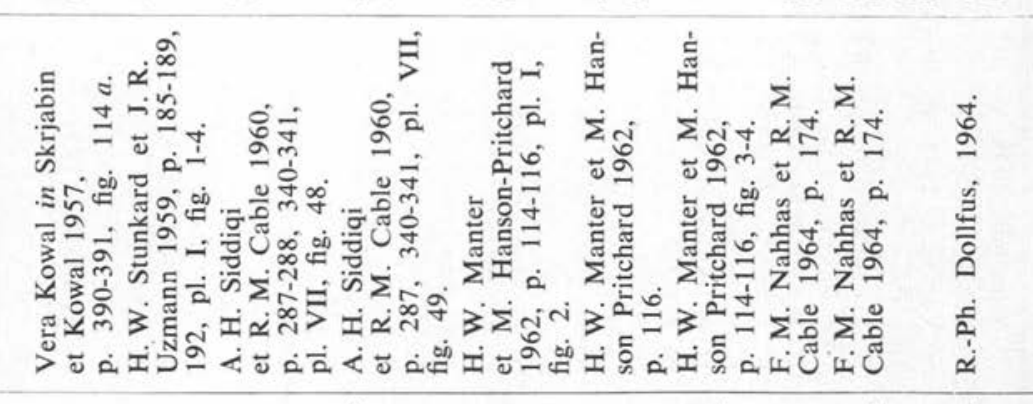 \\
\hline 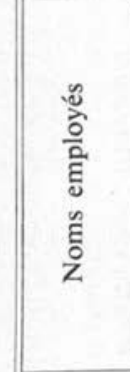 & 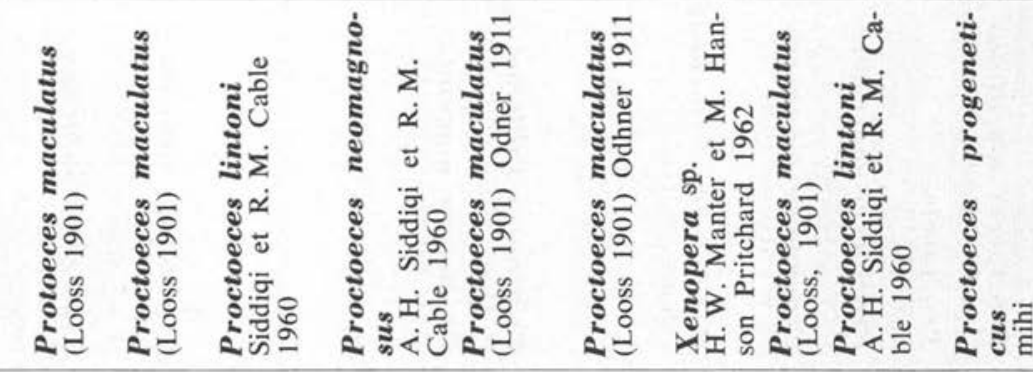 \\
\hline
\end{tabular}


génital s'était allongé d'environ le double de sa vraie longueur. Freeman et Llewellyn ajoutent que quelques-unes de leurs préparations de subtenuis montrent aussi une poche du cirre située presque entièrement en arrière de la ventouse ventrale. Pour ces deux auteurs \& differences in extent of the hermaphrodit duct and the position of the cirrus sac are not in themselves, therefore, sufficient specific characters ».

Qu'il existe des variations individuelles importantes et que des déformations, des déplacements d'organes se produisent lors de la préparation des spécimens, cela est évident; il faut en tenir compte lorsque l'on propose des synonymies, mais la synonymie que j'ai proposée de Proctoeces et Xenopera ne serait pas valable, d'après H. W. Manter et M. Hanson Pritchard (1962, p. 116). Pour ces auteurs, la distinction entre les deux genres se justifie par l'existence d'une vésicule séminale externe chez Xenopera (voir Manter et Hanson Pritchard 1962, p. 114, pl. I, fig. 3 et 4). Ce caractère étant le seul que l'on puisse invoquer pour admettre la validité de Xenopera, il est, à mon avis, insuffisant comme caractère générique et je considère Xenopera comme tout au plus un sous-genre.

\section{LE STADE CERCARIA CHEZ LES FELLODISTOMATIDAE}

Je me limiterai ici aux sous-familles : Steringophorinae, Fellodistomatinae, Tergestiinae, Proctoecinae, Monascinae, Rhodotrematinae, et je dois dire que ma conception de la famille Fellodistomatidae n'est pas celle proposée par R. M. Cable (1953, p. 416) (5), mais celle que j'ai adoptée en 1952 (vide supra).

A) La cercaire est leptocerque (Steringophorinae, Section I). D'après G. K. Chubrik la cercaire de Steringophorus furciger (P. Olsson 1868) est leptocerque à longue queue ; sur la côte mourmane, ses sporocystes parasitent Leda pernula O. F. Müller. La métacercaire que G. K. Chubrik $(1957$, p. 9, 12) considère comme celle de cette espèce a été trouvée par elle, enkystée chez Astarte borealis (Chemnitz 1784), Astarte crenata (Gray 1824), Astarte elliptica (Brown 1827) et Astarte montagui (Dillwyn 1817), de la côte mourmane.

B) La cercaire est trichocerque (Steringophorinae, Section II). Beaucoup de trichocerques, anatomiquement, sont des larves de genres tels que Steringotrema, Bacciger, Lintonium. J'ai personnellement étudié quelques cercaires de ce groupe (voir, en particulier, R. Ph. Dollfus 1911, p. 423, fig. 3 ; 1925, p. 55-60, fig. 5-6).

Le cycle évolutif de Bacciger bacciger (Rudolphi), des Atherina, a été découvert par Ar. Palombi (1933, p. $1-10$, fig. $1-6 ; 1934$, p. $68-72$, fig. $13-16 ; 1934$, p. $438-476$, fig. $1-33 ; 1940$, p. 15-16, fig. 9). La cercaire s'enkyste chez un Amphipode. Il se peut

(5) Pour R. M. Cable (1953, p. 416-417), la famille Fellodistomatidae comprend les sousfamilles Fellodistomatinae (W. Nicoll 1909) emendata, Haplocladinae, T. Odhner 1911, emendata, Tandanicolinae T. H. Johnston 1927, emendata, Gymnophallinae T. Odhner 1905, emendata. Je suis en complet désaccord sur l'admission des Gymnophallinae dans cette famille. Je reviendrai sur ce sujet dans une publication ultérieure.

Au sujet d'une parenté des Fellodistomatidae avec les Gymnophallinae, parenté que j'estime inacceptable, voir R. M. Cable (1952, p. 36-37; 1953, p. 413-414). 
que la cercaire de Bacciger, décrite par N. Latyscheva (1939, p. 220-222, fig. 3-4), soit la même que celle décrite par Palombi. On doit aussi rapporter aux Steringophorinae, Section II, Cercaria pennata D. F. Sinitzin (1911, p. 35-36, 125, pl. V, fig. 76-77) et plusieurs autres, dont celle que W. E. Martin (1945, p. 203-207, pl. I, fig. 1-6) a décrite sous le nom de Cercaria laevicardium W. E. Martin, larve de Lintonium vibex (Edwin Linton), Stunkard et Nigrelli. Cette cercaire a été redécrite sous le nom Cercaria laevicardii R. M. Cable (1954, p. 16-18, pl. I, fig. 1-4), par R. M. Cable, qui a aussi décrit plusieurs autres trichocerques voisines (voir Cercaria caribbea, XXXIX, R. M. Cable 1956, p. 502, 534, 570-571, pl. XIII, fig. 95 ; même figure in Cable 1953, p. 421 , pl. II, fig. 6 ; Cercaria caribbea, LII, R. M. Cable 1963, p. 430-432, fig. 1-2 ; Cercaria caribbea, LIV, R. M. Cable 1963, p. 431, 432-433, fig. 6-7 ; Cercaria caribbea, LV, R. M. Cable 1963, p. 431,433 , fig. 3-5, 8).

C) La cercaire est furcocerque (Fellodistomatinae sensu restricto: intestin long, atteignant ou dépassant le niveau du bord postérieur des testicules). La cercaire est connue pour Fellodistomum fellis (P. Olsson 1868) (6), dont le cycle évolutif a été découvert et étudié à tous les stades par G. K. Chubrik (1952, p. 654-657, fig. 2-8 ; 1954, p. 129). Le stade métacercaire, qui se trouve chez Ophiura sarsi Lütken, n'est pas enkysté. Cette métacercaire, dit Chubrik, est la même que celle trouvée dans l'intestin du même hôte, de la côte mourmane, par A. Tauson (1917, p. 149-218, fig., texte 1-10, pl. V, fig. 1-33) qui la décrivit sous le nom «Adolescaria ophiurae », mais supposa qu'elle correspondait à Steringophorus furciger (P. Olsson 1868).

D) La cercaire est furcocerque (Tergestiinae) comme chez les Fellodistomatinae, mais la morphologie et l'anatomie de l'adulte sont assez particulières pour admettre, avec K. I. Skrjabin et V.P. Koval (1957, p. 167-169, 420), qu'il s'agit d'une sousfamille séparée.

Pour T. Odhner (1911, p. $529 ; 1911$, p. 113), c'est une cercaire de Tergestia qui a été figurée par Ad. de La Valette-Saint-Georges (1855, p. 38, pl. II, fig. 1) ; Cercaria dichotoma Joh. Müller, du plancton de Nice.

J'ai reconnu une cercaire de Tergestia dans une larve planctonique figurée par Saville Kent : Cercaria kenti R. Ph. Dollfus 1927, p. 111-113, fig. 1.

W. A. Haswell (1903, p. 506-507) a décrit une cercaire qu'il avait observée dans * the sporocyst of an Echinostomum » et-a reconnu qu'elle ressemblait à un distome figuré par M. Stossich, disant «probably is the larva of a form nearly related to that species ». Haswell n'a pas eu à sa disposition la figure originale de Stossich, mais la copie par Max. Braun, dans le Bronn's Thier-Reich, pl. XXI, fig. 8, d'une figure donnée par Fr. Sav. Monticelli (1890, pl. XXII, fig. 1), représentant la région antérieure, bien caractéristique, de Tergestia laticollis (Rudolphi 1819), type du genre.

L’appartenance évidente à Tergestia a été soulignée par T. Odhner (1911, p. 529)

(6) Il est incompréhensible que Ben Dawes (1946, p. 203, 242) ait placé Fellodistomum agnotum W. Nicoll 1909 en synonymie de F. fellis (P. Olsson 1868). L'espèce de Nicoll a été retirée du genre Fellodistomum et est devenue Steringophorus agnotus (W. Nicoll 1909), R.-Ph. Dollfus 1952. 
et le matériel original de Haswell a été redécrit par L. Madeline Angel (1960, p. 75-85, fig. 1, 3-5, 7-10).

Une quatrième description de cercaire de Tergestia est celle de Cercaria mathiasi G. Dubois, J. G. Baer et L. Euzet (1952, p. 503-509, fig. 1-5), souvent observée dans le plancton marin de Sète (Hérault), par L. Euzet.

E) La cercaire est furcocerque (Monascinae = Haplocladinae). La cercaire d'une espèce de Monascus (= Haplocladus) a été décrite par W. J. Rees (1947, p. 602-604, fig. 1-2), chez Nucula nucleus (L.) de Plymouth.

Dans un bac de l'aquarium de Kristinberg (Suède) contenant des Nucula nucleus (L.) et des Syndosmya alba (W. Wood 1801), T. Odhner (1911, p. 105) avait trouvé une furcocerque qu'il avait rapportée à Haplocladus minor T. Odhner 1911 ; W. J. Rees ne croit pas que ce soit la même que la sienne, qu'il suppose être, possiblement, celle d'H. typicus T. Odhner 1911 (= Monascus monoenteron A. Looss 1907 et 1912).

On ne sait pas s'il y a enkystement ou non.

F) La cercaire est anoure (Rhodotrematinae : intestin court, n'atteignant pas les testicules ou ne dépassant pas le testicule postérieur). La cercaire est connue pour Rhodotrema quadrilobata Basikalova 1932, dont le cycle évolutif a été découvert et étudié expérimentalement par G. K. Chubrik (1952, p. 981-983, fig. 1-3). La cercaire ne s'enkyste pas, aussi Chubrik a-t-elle considéré le stade métacercaire comme supprimé.

G) La cercaire est microcerque (Proctoecinae). T. Odhner (1911, p. 108, et note $16 ; 1914$, p. 253 , note 1) avait supposé que, en Méditerranée, Cercaria setifera Johan. Müller in Ad. de La Valette Saint-Georges (1855, p. 38, pl. II, fig. II) (non Claparède 1863, nec Villot 1879, nec Monticelli 1888, nec P. Pelseneer 1906) (7) du plancton, à Trieste, pourrait être la larve de Proctoeces maculatus (Looss). Cette supposition fut rejetée par Fr. Sav. Monticelli (1914, p. 35-36).

Cette trichocerque correspond apparemment à quelque espèce de Steringophorinae, mais pas à un Proctoeces. Une cercaire : Cercaria milfordensis J. R. Uzman (1953, p. 445-449, fig. 1-2, pl. II, fig. 5-6), découverte par Uzmann chez Mytilus edulis L., à Mill Neck (Long Island, New York) fut reconnue comme appartenant au genre Proctoeces par Uzmann, qui trouva des larves progénétiques correspondantes dans la masse viscérale de Mytilus edulis L. Dans les œufs de ces larves, le miracidium était mobile et Uzmann estima que l'on était en présence d'un cycle évolutif abrégé.

L'étude de cette cercaire et de la métacercaire correspondante a été continuée à Woods Hole (Massachusetts), par H.W. Stunkard (voir Stunkard et Uzmann 1959, p. 187-193, fig. 1-8, sporocytes, cercaire, juvéniles immatures, individus gravides). Ces

(7) R. M. Cable (1954, p. 18) dit, à propos de C. setifera Müller nec Monticelli \& the larva of Bacciger bacciger according to Palombi 1934 , mais, dans l'ouvrage de Palombi 1934 cité par Cable, $C$. setifera Joh. Müller n'est pas mentionnée par Palombi comme se rapportant à Bacciger ; il n'est pas non plus question de ce rapprochement dans d'autres publications de Palombi. 
auteurs ont rapporté ce matériel à $P$. maculatus (A. Looss), pour eux synonyme de subtenuis.

Ainsi, il y a, dans une même famille, Fellodistomatidae, des cercaires trichocerques, des cercaires leptocerques, des cercaires anoures, des cercaires furcocerques et des cercaires microcerques; mais, comme je le crois, dans une même sous-famille, sauf celle des Steringophorinae, il n'existe qu'une seule sorte de cercaire (8), aussi ai-je retiré Rhodotrema des Fellodistomatinae pour le placer dans une nouvelle sous-famille : Rhodotrematinae.

Il y a des trichocerques dans plusieurs familles, très éloignées entre elles, de même pour les leptocerques, les anoures, les furcocerques et les microcerques. Le caractère trichocerque, ou leptocerque, ou anoure, ou furcocerque, ou microcerque, n'est pas suffisant pour l'attribution d'une cercaire à une famille, il faut connaître d'autres caractères, en particulier la forme et l'extension de la vessie excrétrice et celles de l'intestin. Si je rappelle ici cette vérité première, c'est parce que R. M. Cable (1954, p. 19), voit dans les modalités de l'appendice caudal \& evidence that the Brachylaemidae, Fellodistomatidae and Bucephalidae may form a related group within the Strigeatoidea ».

\section{Bibliographie}

ANGel (L. Madeline), 1960. - Cercaria haswelli Dollfus 1927: a reexamination of Haswell's material, with discussion of the genus Tergestia. Libro Homenaje al Doctor Eduardo Caballero y C., Mexico 1960, p. 75-86, fig. 1-10.

Caballero y C. (Eduardo), 1959. - Consideraciones en torno a la familia Xenoperidae Poche, 1925 (Trematoda, Digenea Carus 1863). Revista Biologia tropical, v. VII, n. 1,1959 , p. $57-61$.

Cable (Raymond M.), 1952. - Studies on marine digenetic trematodes of Puerto Rico. The systematic position of the subfamily Gymnophallinae Odhner. Journ. Parasitology, v. XXXVIII, n. 4, section 2, august 1952, p. 36-37.

-, 1953. - The life cycle of Parvatrema borinqueñae gen. et sp. nov. (Trematoda: Digenea) and the systematic position of the subfamily Gymnophallinae. Journ. Parasitology,

v. XXXIX, n. 4, section 1, august 1953, p. 408-421, pl. I, fig. 1-5, pl. II, fig. 6-8.

-, 1954. - A new marine cercaria from the Woods Hole region and its bearing on the interpretation of larval types in the Fellodistomatidae (Trematoda: Digenea). Biolog. Bull., Woods Hole, v. CVI, n. 1, 15-20 febr. 1954, p. 15-20, pl. I, fig. 1-4.

-, 1956. - Marine Cercariae of Puerto Rico. Scientific Survey of Porto Rico and the Virgin Islands, New York, v. XVI, Part 4, 26-4-1956, p. 489-577, pl. I-XVI, fig. 1-117.

(8) Si l'on accepte, comme le propose R. M. Cable (1939, p. 414), d'inclure dans les Fellodistomatinae la sous-famille Tandanicolinae T. H. Johnston (1927, p. 136), il faudra ajouter aux diverses formes de cercaires que l'on connaît déjà dans la famille, les trichofurcocercaires, représentées par "Cercaria (Furcocercaria) trichofurca », T. H. Johnston et L. M. Angel (1940, p. 331-334, fig. 1-7), larve de Tandanicola brancrofti T. H. Johnston 1927. Ainsi, à chaque sous-famille de Fellodistomatidae, correspond une forme différente de cercaire. 
-, 1963. - Marine Cercariae from Curaçao and Jamaica. Zeitschrift für Parasitenkunde, Bd XXIII, $\mathrm{n}^{\circ} 3$, abgeschlossen, 2-12-1963, p. 429-469, fig. 1-57.

Chubrik (= Tchoubrik) (G.-K.), 1952. - Stade larvaire du Trématode Fellodistomum fellis Nicoll 1909 chez une Ophiure de la mer de Barents. Zoologhitcheskii Journal, v. XXXI, livr. 5, 1952, p. 653-658, fig. 1-8.

-, 1952. - Cycle évolutif de Rhodotrema quadrilobata Basikalova, parasite des poissons pleuronectes. Doklady Akad. Nauk SSSR, v. LXXXIII, n. 6, 1952, p. 981-983, fig. 1-3.

-, 1954. - Parasitological Investigations of Mollusca of the littoral zone of Murmansk and the White Sea. Trudy Problemnich $i$ Tematicheskich Eoveshanii, livraison 4, Zool. Inst. Akad. Nauk., 1954, p. 128-134, fig. 1-2.

—, 1957. - Parthénites et larves de Trématodes des Mollusques de la mer Blanche et de la Mourmanie orientale. Résumé d'auteur d'une dissertation pour l'obtention du grade universitaire de Candidat en Sciences biologiques. Edition de l'Université nationale A.A. Jdanov, de Léningrad, 1957, 21 p.

Dawes (Ben), 1946. - The Trematoda with special reference to British and other European Forms. Cambridge University Press, 1946, xvi + 644 p., fig. 1 A-81 H.

-, 1947. - The Trematoda of British Fishes. Ray Society, London, n. 131, 11 april 1947, $\mathrm{VIII}+364$ p., fig. 1 A-51 F.

Dollfus (Robert-Ph.), 1911. - L'appareil néphridien de deux cercaires parasites de Donax vittatus Da Costa. Comptes rendus hebdom. séances Soc. Biologie, Paris, v. LXXI, n. $31,17-11-1911$, p. $422-425$, fig. 1-4.

—, 1925. - Liste critique des cercaires marines à queue sétigère signalées jusqu’à présent. Travaux de la Station zoologique de Wimereux, v. IX, Paris, 1925, p. 13-55, fig. 1-6.

-, 1925. - Voir Ts. Fujita.

—, 1927. - Sur une larve planctonique figurée par Saville Kent. Notules sur des cercaires atlantiques. Bull. Soc. zool. France, v. LII, n 2, séance du 8-3-1927, p. 111-114, fig. 1.

-, 1952. - Miscellanea helminthologica maroccana, IV. Affinités naturelles de Pseudochetosoma salmonicola $\mathrm{R}$.-Ph. Dollfus 1951 (Famille Steganodermatidae nov.). Emendation de la superfamille Haploporoidea W. Nicoll 1935. Arch. Inst. Pasteur Maroc, Casablanca, v. IV, cahier 5, mai 1952, p. 369-386, fig. 1 .

Dubois (Georges), BAER (Jean-G.) et EuZet (Louis). - Une nouvelle cercaire du plancton marin de Sète, Cercaria mathiasi n. sp. Revue suisse de Zoologie, v. LIX, $\mathrm{n}^{\circ} 26$, déc. 1952, p. 503-510, fig. 1-5.

Freeman (R. F. H.) and Llewellyn (J.). - An adult Digenetic Trematode from an invertebrate host: Proctoeces subtenuis (Linton) from the Lamellibranch Scrobicularia plana Da Costa. Journ. marine biol. Association United Kingdom, v. XXVII, 1958, p. 435-457, fig. texte 1-2, pl. I, fig. 1-5, pl. II, fig. 1-7.

Fujra (Tsunenobu), 1925. - Etude sur les parasites de l'huître comestible du Japon : Ostrea gigas Thunberg. Traduction accompagnée de notes, de diagnoses et d'une bibliographie par R.-Ph. Dollfus. Annales Parasitol. hum. et comp., v. III, $\mathrm{n}^{\circ}$ 1, 1-1-1925, p. $37-59$, fig. 1-7. 
Hanson (Mary Louise), 1950. - Some digenetic Trematodes of marine fishes of Bermuda. Proceed. helminthol. Soc. Washington, v. XVII, n. 2, july 1950, p. 74-89, fig. 1-9.

Haswell (W. A.), 1903. - On two remarkable sporocysts occuring in Mytilus latus, on the coast of New Zealand. Proceed. Linnean Soc. New South Wales, 1902, part 4, april 9, 1903, p. 497-515, pl. XIX, fig. 1-15, pl. XX, fig. 16-36.

Johnston (T. Harvey), 1927. - New Trematodes from an australian Siluroid. Transact, royal Soc. South Australia, v. LI, 1927, p. 129-136, fig. texte A (1-4)-B (1-5).

- et ANgel (L. Madeline), 1940. - Larval Trematodes from australian freshwater Molluscs. Part VII. Transact. royal Soc. South Australia, v. LXIV, n. 2, 20-12-1940, p. 331339, fig. 1-15.

LatyscheVA (N.), 1939. - Parasites of some invertebrates of the Azoff-sea. A study connected with the introduction of different Invertebrates from the Azoff-sea into the Caspiansea. Bulletin scientifique de l'L.G.U., 1939, n. 43, p. 213-232.

La Valette Saint-Georges (Adolphus J.-H. de), 1855. - Symbolae ad Trematodum evolutionis historiam. Dissertatio inauguralis, Berolini $14-4-1855$, p. 1-40, pl. I, fig. I-XV + A-S, pl. II, fig. I-VII + A-K.

Linton (Edwin), 1907. - Notes on parasites of Bermuda fishes. Proceed. Un. St. Nat. Museum, v. XXXIII, n. 1560,14 oct. 1907 , p. 85-126, pl. I-XV, fig. 1-102.

Looss (Arthur), 1901. - Ueber einige Distomen der Labriden des Triester Hafens [I]. Centralbl. für Bakt. Parasitenkunde, I. Abt., Bd XXIX, n. 9, 21-3-1901, p. 398-405, fig. 1-4.

MANTER (Harold W), 1940. - Digenetic Trematodes of Fishes from the Galapagos Islands and the neighboring Pacific. Allan Hancock Pacific Expeditions, v. II, n. 14, 27-31940, p. I-v + 329-497, pl. XXXII-XL, fig. 1-136.

-, 1947. - The Digenetic Trematodes of marine fishes of Tortugas, Florida. The american Midland Naturalist, v. XXXVIII, n. 2, sept. 1947, p. 257-416, fig. 1-152.

-, 1954. - Some Digenetic Trematodes from Fishes of New Zealand. Transact. royal Soc. New Zealand, v. LXXXII, part 2, sept. 1954, p. 475-568, fig. 1-89.

- et Hanson Pritchard (Mary), 1962. - Studies on digenetic Trematodes of Hawaiian fishes: families Fellodistomatidae, Opistholebetidae and Gyliauchenidae. Trans. american microscop. Soc., v. LXXXI, $\mathrm{n}^{\circ} 2$, april 1962, p. 113-123, pl. I, fig. 1-6, pl. II, fig. 7-11.

MarTin (W. E.), 1945. - Two species of marine cercariae. Transact. american microscop. Soc., v. LXIV, n. 3, july 1945 , p. 203-212, pl. I, fig. 1-7, pl. II, fig. 8-11.

MoNTICELli (Francesco Saverio), 1914. - Ricerche sulla Cercaria setifera di Joh. Müller. Atti della R. Accad. delle Scienze fis, e mat. di Napoli, v. XV, ser. 2, n. 11, 24-11914, separat. 48 p., fig. texte 1-7, pl. I-V, fig. 1-95.

NahHas (F. M.) and Cable (Raymond M.), 1964. - Digenetic and Aspidogastrid Trematodes from marine fishes of Curaçao and Jamaica. Tulasne University Studies, v. XI, n. 5, 25-5-1964, p. $168-226$, fig. $1-52$.

Nicoll (William), 1915. - The Trematode parasites of North Queensland, III. Parasites of Fishes. Parasitology, v. VIII, n. 1, june 25, 1915, p. 22-41, pl. IV, fig. 1-8, pl. V, fig. 9-11. 
ODHNER (Theodor), 1911. - Zum natürlichen System der digenen Trematoden. III. Zoolog. Anzeiger, Bd XXXVIII, n. 4, 8-8-1911, p. 97-117, fig. 1-8.

-, 1911. - Zum natürlichen System der digenen Trematoden. IV. Zoolog. Anzeiger, Bd XXXVIII, $\mathrm{n}^{\circ} 24,5-12-1911$, p. 513-531, fig. 1-2.

-, 1914. - Cercaria setifera von Monticelli. Die Larvenform von Lepocreadium album Stoss. Zoologiska Bidrag från Uppsala, Bd III, 7-7-1914, p. 247-255, pl. VI, fig. 1-6.

PAlombi (Arturo), 1933. - Cercaria pectinata Huet e Bacciger bacciger (Rud.). Rapporti genetici e biologia. Bollettino di Zoologia, anno IV, n. 1, febr. 1933, p. 1-11, fig. 1-6.

-, 1934, BACCIGER BACCIGER (Rud.). - Trematoda digenetica: fam. Steringophoridae Odhner. Anatomia, sistematica e biologia. Publicazioni della Stazione zoolog. Napoli, v. XIII, fasc. 3,1934 , p. $438-478$, fig. 1-33.

-, 1934. - Gli stadi larvali dei Trematodi del Golfo di Napoli. $1^{\circ}$ Contributo alla studio della morfologia, biologia e sistematica delle cercarie marine. Publicazioni dell Stazione zoolog. Napoli, v. XIV, fasc. 1, 1934, p. 51-94, fig. 1-34.

—, 1940. - Gli stadi larvali dei Trematodi del Golfo di Napoli. $3^{\circ}$ Contributo alla studio della morfologia, biologia e sistematica delle cercarie marine. Revista di Parassitologia, v. IV, n. 1, marzo 1940, p. 1-25, fig. 1-13.

Pogorelseva (T.-P.), 1952. - Matériaux pour la parasitofaune des poissons du Nord-Est de la Mer Noire. Travaux de l'Institut zoologique de l'Acad. des Sciences de l'U.R.S.S., 1952, p. 100-120.

REES (W. J.), 1947. - A cercaria of the genus Haplocladus from Nucula nucleus (L.). Journ. marine biolog. Assoc. United Kingdom, v. XXVI, june 1947, p. 602-604, fig. 1 AB-2.

SidDiQI (Ather H.) and CABLE (Raymond M.), 1960. - Digenetic Trematodes of marine fishes of Porto-Rico. Scientif. Survey Porto-Rico and Virginia Isl., New York, v. XVII, part. 3, July 15,1960 , p. $257-369$, fig. texte $1-2$, pl. I-XXI, fig. 1-139.

SinITZIN (Dmitri Feodorovitch), 1911. — La génération parthénogénétique des Trématodes et leur descendance dans les Mollusques de la Mer Noire. Mém. Acad. impériale Sciences St-Pétersbourg, série VIII, Classe physico-mathém., v. XXX, n. 5, 1911, p. 1-127, fig. texte 1-17, pl. I-VI, fig. 1-100.

SkrJabin (Constantin Ivanovitch) et Koval (Vera P.), 1957. - Famille Fellodistomatidae Nicoll 1913. Trématodes des Animaux et de l'Homme. Bases de la Trématodologie. Edition de l'Académie des Sciences, Moscou, v. XIII, 1957, p. 163-452, fig. 63-129.

- et Guschanskaja (L. X.), 1958. - Sous-ordre Azygiata La Rue 1957. Trématodes des Animaux et de l'Homme. Bases de la Trématodologie. Edition de l'Académie des Sciences, Moscou, v. XIV, p. 667-819, 933, fig. 186-219.

StunKard (Horace W.) et Uzmann (Joseph R.), 1959. - The life-cycle of the Digenetic Trematode, Proctoeces maculatus (Looss, 1901) Odhner, 1911 (Syn. P. subtenuis (Linton, 1907) Hanson, 1950), and description of Cercaria adranocerca n. sp. Biolog. Bulletin, Woods Hole, v. CXVI, n. 1, febr. 1959, p. 184-193, pl. I, fig. 1-4, pl. II, fig. 5-10.

TAuson (A.), 1917. - Adolescaria ophiurae, parasite d'Ophiura sarsi Lütken. Journal russe de Zoologie, Pétrograde. Zoologitcheskii Vestnik, v. II, n. 1-2, 1917, p. 149-218, fig. texte $1-10$, pl. V, fig. 1-33. 
Uzmann (Joseph R.), 1953. - Cercaria milfordensis nov. sp., a microcercous Trematode larva from the marine bivalve, Mytilus edulis L., with special reference to its effect on the host. Journ. Parasitology, v. XXXIX, n. 4, section 1, august 1953, p. 445 451, pl. I, fig. 1-2, pl. II, fig. 3-6.

Winter (Howard A.), 1954. - Proctoeces macrovitellus nov. sp., de un pez embiotocido del Océano Pacifico del Norte. Ciencia, Mexico, v. XIV, n. 7-8, 15-12-1954, p. 140142, fig. 1-2.

WlassenKo (P.), 1931. - Zur Helminthenfauna der Schwarzmeerfische. Travaux de la Station biologique à Karadagh, Livre 4, 1931, p. 88-136, fig. 1-26.

Yamaguti (Satyu), 1934. - Studies on the Helminth Fauna of Japan. Part 2. Trematodes of Fishes, I. Japan. Journ. Zoology, v. V, n. 3, march 31, 1934, p. 249-541, fig. 1-145.

-, 1938. - Studies on the Helminth Fauna of Japan. Part 21. Trematodes of Fishes IV. Kyoto, march 10 -april 10,1938 , p. 1-139, fig. 1-83, pl. fig. 1-9.

-, 1940. - Studies on the Helminth Fauna of Japan. Part 31. Trematodes of Fishes VII. Japan. Journ. Zoology, v. IX, n. 1, 1940, p. 35-108, fig. texte 1-50, pl. I, fig. 1-8, pl. II, fig. 9-12.

Institut Scientifique Chérifien, Rabat. Muséum National d'Histoire Naturelle, Paris 